\title{
Sachregister
}

Abbildungsfunktion 56

Abstraktion 56, 107

Abstraktion, genetische 53

Abstraktionsverfahren 59

Académie des Sciences 218

Académie française 281

Accademia della Crusca 217, 281

Akademie 217, 281, 284, 319-320

Alchemie 178

Alltagswelt 514

Analogie 17, 119-120, 123, 128, 143, 253, 362, 423, 445

Analyse $10,74,81,122$

Analytiker 26

Anastomose 22, 62

Anatomie, vergleichende 35, 39

Angemessenheit 587

Anglizismus 288

Anthropomorphismus 10, 111, 147

Anweisungsstil 178

Appell 116

Appellfunktion 490

Apperzeptionsgewohnheit 160

Apperzeptionsverweigerung 300, 448

Arabisch 178

artes liberales 171, 177-178, 213

artes magicae 172, 225

artes mechanicae 172, 178, 225

Attraktion 430

Aufklärung 279, 285, 287

Ausdehnung 106

Ausdruck 115

Ausdrucksfunktion 490

Austausch der Denkstile 566

Autopoiesis 11

Autoritätenkanon 173

Basisfunktionen der Sprache 485, 493, 581

Baum des Lebens 504

Bedeutung 45, 100

Bedeutungsveränderung 197

Begriffs-Realismus 72

Begriffsbildung 27, 38

Begriffsbildung, klassifikatorische 47
Begriffsbildung, topologische 47

Begriffsschema 113

Berliner Sozietät/Akademie der Wissenschaften $223,284,286,564$

Bibelsprache 267

Bild 530

Bildersprache 305

Bildstil 509, 516

Bildtyp 527

Bildung 73, 351, 353

Bildungssprache 162, 176, 348, 554, 567, 579

Bildungstrieb 107-108, 111, 342

Bilingualismus 261, 323, 568

Binnengliederung der Sprache 97

Biographie 95

Biologie 183, 423

Blatt 57

bon usage 276,320

Botanik 327

Buchdruck 239

Buchmarkt 229

Buchmetaphorik 253

Buchproduktion 210, 277

Chemie 188, 295, 297, 299, 301, 305, 308, 327,356

Chronik 95

Darstellung 115, 485

Darstellung, dogmatische 89

Darstellung, genetische 89

Darstellung, systematisch-genetische 102

Darstellung, wissenschaftliche 175

Darstellungsmodelle 344

Darstellungsmuster 96, 347

Darstellungstechnik 416

Darstellungstypus 97, 485

Darstellungsverfahren 27

Debatte, öffentliche 582

deduktiv 66

Definition 29, 34, 184, 340, 422, 488

Demokratisierung 472

Demokratisierungsparadoxon 540

Ә Open Access. () Jürgen Schiewe, publiziert von De Gruyter. (c) BY-NC-ND Dieses Werk ist lizenziert unter der Creative Commons Attribution-NonCommercial-NoDerivatives 4.0 Lizenz.

https://doi.org/10.1515/9783110692716-025 
Denkmodell 346, 348, 367-368, 371, 429, 530

Denkmodelle, soziomorphe 365

Denkstil 261, 266, 329-330

Deutsch 166, 168, 216, 222, 256, 258-259, $266,321,330,462,466,468,507,557-$ $558,562,567$

Deutschland 221, 277

Dichter 84

Dichtkunst 78

Dichtung 286

Diglossie 324, 568

Diglossie, sprachinterne 259, 266

Doppel-Helix 496, 510, 515, 517-518, 525

Dreieck, semiotisches 100

Einbildungskraft 24

Einschachtelung 103

Embranchement 131

Empirie 162

Empirismus 72

England 221

Englisch 166, 168, 199, 462, 466, 471, 473, 557-558, 563, 569-570

Entlehnung 193-195, 278, 394, 465, 471, 553

Entlehnungsschub 192, 224

Entscheidungsrede 585, 587

Epigenese 12, 103, 108, 112, 338

Erfahrung 76-81, 89-90, 339

Erkenntniskritik 337

Erklärungsverfahren, etymologisches 178

Erweiterung des gegebenen Horizonts 489, 499

Etymologie 282

Euphemismus 198

Evolution 338

Evolutionslehre 190, 393, 395

Expansion 430

Fachausdruck 578

Fächersystematik 212

Fachjargon 543

Fachprosa 190, 356, 475

Fachsprache 288, 419, 475

Fachsprachen, naturwissenschaftliche 171, 174

Fachstil 199
Fachvokabular 173, 258-259, 326, 483

Fachwerksprache 257

Fachwerkstil 255, 259, 261, 266, 324-325, 327,568

Fachwissenschaft 231

Farbenlehre 12, 66, 299, 343, 350

Farbwörter 67

Form 114

Form, autobiographische 88

Fortpflanzung 63

franglais 275

Frankreich 220, 275

Französisch 463, 469, 564-565

Fremdwort 282, 442, 463, 473, 554

Fremdwörterstil 421

Fruchtbringende Gesellschaft 278

Funktion der fragenden Erweiterung des Horizonts 581

Funktion, Appell 581

Funktion, Ausdruck 581

Funktion, Darstellung 581

Funktion, Kontakt 581

Funktion, metasprachliche 581

Funktion, poetische $115,493,581$

Funktion, Unterscheidung 581

Funktionalstil 174

Funktionswandel der Wissenschaft 566

Gattung 5, 116, 347-348, 374, 447, 459, 484

Gebrauchsliteratur, praktische 206

Gegenstand 45

Gegenwirkung 383, 385

Geisteswissenschaft 203, 353,541, 545

Gelehrte 277

Gelehrtenlatein 4, 177, 202, 213, 229

Gelehrtensprache 172

Gemeinsprache $170,175,288$, 415, 419, 426, 434, 442, 475, 487, 582

Gemeinverständlichkeit 274

Gen 495

Generalisierungslücke 63

Genese der Pflanzen 55

Germanistik 204

Gestalt 73

Gliederung 486

Griechisch 149 
Handbuchwissenschaft 176, 497, 499, 513, 526

Hervorbringen 103

Herzog August Bibliothek Wolfenbüttel 215

Hierarchien, lexikalische 33

homo monolinguis 467

Homöopathie 373-374, 380, 382, 385-386

honnête homme 208, 276, 320

Hypostasierung 109, 135

Hypothese 513

Idealismus 72

Idee 48, 76-79, 81, 89-90, 339

Idol 495, 515-516

induktiv 66

Italienisch 205

Jargon 440

Kampf ums Dasein 10, 190-191, 365, 392, 401-404, 580

Klassifikation 29

Klassik 494

Kommunikationsdreieck 153

Komposition 130

Konnotat 580

Kontakt 116

Kontaktfunktion 489

Kontinuum 53, 61

Kontinuum, heterogenes 339,580

Koran 323

Kritik an der Sache 538

Kritik an der Sprache 538

Kultur 195

Kultur, europäische 334

Kunst 86

Kunstausdruck 23, 28

Kunstwörter 21

Laien 277

Landessprache 219, 230, 261, 311, 316, 329

Latein 149, 156, 158, 168, 205, 210, 216, 219 , 222, 232, 234, 255, 258-259, 261, 266, $277,279,283,285,288,310-312,316$, $324,329-330,334,355,390,468,475$, 562,565

Lebenskraft 107, 342, 385
Lehnbildung 233, 314, 563

Lehnprägung 234, 255, 263, 266, 316-317

Lehnübersetzung 180, 263

Lehnwort 263

Lehrbuchwissenschaft 176

Lehrbuchzeichnung 517

Lehrerausbildung 547

Lehrvortrag, anatomischer 95

Leopoldina 576, 582

Licht 133

Linguistik 192, 541, 554, 560

Linguistik, germanistische 544-545

Linguistik, moderne 1

Literatur 447, 473

Magnetismus 139

Matériaux 129

Materialismus 72

Mathematik 162, 212-213, 227, 300, 327

Medizin 261

Mehrsprachigkeit 557, 571

Merkmale, relationale 65

Merkmale, semantische 33

Messkatalog 213

Metamorphose 60, 64, 102, 112

Metapher 10, 42, 110, 128, 132, 192, 361, 369-371, 394, 400, 409, 412, 425, 428, 445, 449, 459, 488, 502, 504, 519, 525 , 529-531, 553

Metaphernkritik 10

Metaphorik 111, 127, 136, 183, 355, 390, 407, 553

Metaphorik, anthropomorphe 371

Metaphorik, soziomorphe 371

Metasprache 116, 186, 487

Methode, dogmatische 98,115

Methode, genetische 98, 115

Methode, morphologische 74

Mischprosa 177

Mischsprache 257, 261, 266, 323, 325

Missbildung 64

Morphologie 73

Mündlichkeit 269

Name 45-46, 369

Namenbündelung 45, 530

Nationalsprache 234, 237 
Natur 15, 25, 81, 83, 85-86, 93, 99, 384

natural preservation 398

natural selection $10,190,364,372,395,398-$ $399,412,562,580$

Natürliche Zuchtwahl 10, 190-191, 365, 402, 580

Naturphilosophie 138, 141, 143, 163, 177, 204, 316, 383

Naturreiche 14, 16, 20, 25, 28-29, 35, 86, 102, 117,361

Naturwissenschaft $86,181,203,225,248$, $303,309,350,353,355,390,405-406$, 424, 429, 431, 466, 495, 514, 545, 554, 559-560, 573-574, 583

Naturwissenschaftssprache $170,557,562$, 577,580

Neubildung 181

Neutralität, sprachliche 499

Nomenklatur 62, 68, 188, 296, 306, 485

Nomenklatur, binäre 33,184

Nominalismus 72, 249

Norm 278, 315

Norm, soziale 315, 539

Objekt 71

Optik 66, 300

Orangenstil 91

Ordnungsform 168

Ordnungsverfahren 25

Organ 57

Orientierungsverzicht 406

Paarformel 259

Pädagogik 542

Paradigmenwechsel 302, 513

Paraphrase 488

Pflanzenreich 85

Phlogiston 291-292, 294-296, 300,302, 306

Phlogistontheorie 151, 188

Physik 80, 186, 213, 327, 424

Pléiade 276

Plan 132

Plastikwort 514

Poesie 158, 160, 162, 200

Polarität 102, 141

Politikberatung 574, 576, 582
Popularisierung 196, 200, 202, 288

Populärwissenschaft 176, 231, 406, 498-499, 513, 526, 566

Postversandbuch 479

Prädikation, definierende 59

Praeidee 515

Praeidol 515-516

Präformation 11-12, 108, 112

Präformationslehre 103

Predigttradition 84

Prestige 198

Prosa, populäre 198

Prosastil 286

Psychoanalyse 192, 368-369, 409-411, 414, 424, 430, 435, 439, 454

Psychologie 191

Publikum 211, 239, 280

Publikumsorientierung 479, 493

Purismus 274

qualitas occulta 109

Rationalismus 72

Rätsel 458

Realismus 72

Referendarausbildung 549

Referenz 45, 100

Regelverstoß 155, 169

Reihe 70

Reihe, lockere 99, 102, 115, 346-347

Reihe, semantische 113, 122, 341, 343

Reihe, stetige 70,102, 343

Relativitätstheorie, sprachliche 67

Rhetorik 158, 200, 266, 272, 280, 313, 585

Richtigkeit 20, 443-444

Rollenwort 248

Royal Society 218,320

Rückübertragung 13, 191, 363, 368, 371, 402, 514

Sachbuch 200, 475-476, 491, 581

Sachbuch, populärwissenschaftliches 479

Sachliteratur 203, 473, 481, 489, 492, 551

Sachliteratur, populäre 289

Sachorientierung 493 
Sachprosa 170, 177, 179, 182, 201, 204, 209, 224, 233-234, 238, 242, 256, 279-280, 286, 290, 314, 320-321, 470, 475, 494, 565

Sachprosa, populäre 492

Sachsteuerung 197, 366, 370, 400, 440

Säkularisierung 190

Scheinfachlichkeit 552

Schema 113, 342

Schema, hypothetisches 517

Schlagwort 190, 365, 396

Schlüssel (clavis) 51, 184

Schlüsselbegriff 514

Schraube 520-522, 528, 531

Schriftsprache 286, 312

Schweden 222

Sehen 9

sermo humilis 271, 273

Sexualität 435, 444

Sinn 45, 100

Skizze, hypothetische 516

social borrowing 193, 394, 431

Sozialdarwinismus 11, 191, 366

Sozialwerkzeug 518

Sozialwissenschaft 554

Sphärenvermengung 110, 117, 120, 123-124, 127, 133-135, 143-144, 147, 346, 361, 363, 407, 411, 446

Spirale 519, 521-522, 526, 531

Spiritualismus 72

Sprachausbau 473

Sprachbegriff 261, 274

Sprachbewusstsein 43

Sprachdeterminismus 400

Sprachdifferenzierung 186, 289

Sprache 6, 14, 21, 24, 68, 80, 127, 133, 139, 144-145, 147, 149-150, 153-154, 167, 250, 261, 299, 337, 362, 371, 412, 448, 467, 506, 519, 521, 529

Sprache, anthropomorphe 359

Sprachenbaum 505

Sprachenkontakt 470

Sprachenstreit 202

Sprachentrennung 222, 321

Sprachenwechsel 261, 566

Sprachfassade 543

Sprachfunktionen 115, 578
Sprachfunktionen-Modell 176

Sprachgebrauch 312, 467, 539

Sprachgebrauchstradition 169

Sprachgeschichte 310, 434, 561, 565

Sprachgeschichtsschreibung 234, 317

Sprachgesellschaften 179, 209, 279, 283, 320,565

Sprachideale 282, 290

Sprachkrise 209, 279, 321

Sprachkritik 274-275, 277, 288, 290, 299, 338, 434-435, 537, 553-554, 565

Sprachkritik, aufklärerische 286

Sprachkritik, publizistische 537

Sprachkultur 286, 290

Sprachmengerei 179, 209, 222, 278-279, 321, 564

Sprachplanung 319

Sprachprägung 299

Sprachproduktion, technisierte 289

Sprachpurismus 150

Sprachreflexion, erkenntniskritische 362

Sprachreflexion, erkenntnispraktische 362

Sprachskepsis 139, 337

Sprachsphären 126

Sprachstil 261, 426, 516

Sprachtheorie 43

Sprachtyp 75, 460

Sprachunterricht 473

Sprachverhaltenstypus 316

Sprachwandel 466

Sprachwissenschaft 275, 430, 537-538

Sprachwissenschaft, historische 1

Sprachwissenschaft, inhaltbezogene 153

Sprechen, hypothetisches 116

Staat 195

Stadt 157

Stammbaum 500, 502, 515, 517

Standardisierung 472

Standardsprache 470,472

Standardsprache, deutsche 286

Stickstoff 295

Stil 283, 312

Stil, mündlicher 270

Stil, wissenschaftlicher 84

Stildifferenzierung 196

Stilgrammatik 324

Stilideal 159, 177 
Stoff 114

struggle for life $10,190-191,364,372,395-$ $397,412,514,562,580$

Subjekt 71

Surrogat 137

Symbolik 139, 144

Symbolik, universelle 117

Synonyme 46

Synonymenvariation $43,48,102,128,258$, $344,348,488,530$

Synthese $10,23,74,81,122$

Synthetiker 26

System 315

\section{Tatsache 497}

Taxonomie 27, 184

Terminologie $64,68,123,174,180,250,252$, $307,338,350,414,417,424,428$, 441442, 487

Terminologie, physikalische 187

Terminologietypen 325, 327

Terminus 20, 44, 100, 102-103, 106, 180, 231, $282,307,316,333,370,400,410,416$, 419-421, 439, 483, 485, 514, 580

Textsorten 97, 99

Textsortenattrappe 440

Textsortenübertragung 438

Textsortenverschränkung 427

Theologie 213

Theorie der Sprache 14

Theoriesprache 176

Totalität 351

Typus $39-44,46,50,53,79,102,344$

Übersetzungsarbeit 483

Umgangssprache 176, 202, 231-232, 397, $541,550,552,573,576,580$

Umgangssprache, fachliche 176

Universalbegriff 552

Universalsprache 182, 209, 233, 284, 311, 462

Universität 157, 171, 208, 212, 229, 259, 261, 277, 284-285, 329-331, 540, 566, 575

Unverständlichkeit 578

Urpflanze 52, 336

Urphänomen 69,71, 102

usage 539
Variationsstil 42

Veranschaulichung 509

Veranschaulichung, populäre 517

Verdeutschung 287

Verfachlichung 537

Verfahren, analogisierendes 363

Verfahren, analytisches 363

Verfahren, deduktives 486

Verfahren, induktives 486

Verfahren, synthetisches 363

Vergleichen 125

Verschriftlichung 551

Verstehen, wortgesteuertes 366

Verteilersprache 176

Verwaltungssprache 548

Verwissenschaftlichung 196, 200, 537, 541, 550, 555

Vielsprachigkeit 555

Visiotyp 496, 510, 514-515

Visiotyp, globales 513

Visiotyp, universelles 516-517

Visualisierung 519, 521

Volksgeist 274

Volkssprache 279, 288, 355, 390, 475

Vorlesung 156

Vorlesungssprache 329

Vorstellungsart 105, 123, 149, 304-305, 338, 346,351

Vulgata 323

Wahlverwandtschaften $123,188,340,356-$ $358,365,369-370,398-399,409$

Wahrheit 443

Welt, besprochene 88

Welt, erzählte 88

Weltansichten 149, 571

Wissen 248

Wissenschaft 158, 162, 406, 465

Wissenschaftsgeschichte 154, 167, 303, 308, 447

Wissenschaftsparadigma 513

Wissenschaftsproduktion 546

Wissenschaftssprache $1,115,176,355,439$, $466,475,543,545,555,568,576,582$

Wissenschaftssprache, deutsche 210, 222, $229,279,283,285,321,328,564,572$

Wissenschaftstheorie 308 
Wissenschaftswerkzeug 518

Witz 24

Wort 7, 100, 111, 281, 495, 529

Wörterbucharbeit 179, 209, 223, 281

Wörterbuchprogramm 321, 565

Wortreihe 552

Wortschatz 281

Wortschatzerweiterung 194

Wortsteuerung 198, 400, 441

Zeichen 462

Zeichen, sprachliches 249, 281, 293-294, 370,410
Zeichen, visuelles 515, 518, 529

Zeichenterminus 306

Zeichnung 6

Zeitschriftenwissenschaft 176, 497, 525

Zoologie 327

Zusammenziehung 106

Zweisprachigkeit 468,568

Zwillingsformel 259

Zwischenkieferknochen 36, 47

Zwischenwelt, sprachliche 299,552 